\title{
Meta-analyses of genome wide association studies in lines of laying hens divergently selected for feather pecking using imputed sequence level genotypes
}

Clemens Falker-Gieske ${ }^{1 *}$ (D), Hanna Iffland ${ }^{2}$, Siegfried Preuß ${ }^{2}$, Werner Bessei ${ }^{2}$, Cord Drögemüller ${ }^{3}$, Jörn Bennewitz ${ }^{2}$ and Jens Tetens $s^{1,4}$

\begin{abstract}
Background: Feather pecking (FP) is damaging behavior in laying hens leading to global economic losses in the layer industry and massive impairments of animal welfare. The objective of the study was to discover genetic variants and affected genes that lead to FP behavior. To achieve that we imputed low-density genotypes from two different populations of layers divergently selected for FP to sequence level by performing whole genome sequencing on founder and half-sib individuals. In order to decipher the genetic structure of FP, genome wide association studies and meta-analyses of two resource populations were carried out by focusing on the traits 'feather pecks delivered' (FPD) and the 'posterior probability of a hen to belong to the extreme feather pecking subgroup' (pEFP).

Results: In this meta-analysis, we discovered numerous genes that are affected by polymorphisms significantly associated with the trait FPD. Among them SPATS2L, ZEB2, KCHN8, and MRPL13 which have been previously connected to psychiatric disorders with the latter two being responsive to nicotine treatment. Gene set enrichment analysis revealed that phosphatidylinositol signaling is affected by genes identified in the GWAS and that the Golgi apparatus as well as brain structure may be involved in the development of a FP phenotype. Further, we were able to validate a previously discovered QTL for the trait pEFP on GGA1, which contains variants affecting NIPA1, KIAA1211L, AFF3, and TSGA10.
\end{abstract}

Conclusions: We provide evidence for the involvement of numerous genes in the propensity to exhibit FP behavior that could aid in the selection against this unwanted trait. Furthermore, we identified variants that are involved in phosphatidylinositol signaling, Golgi metabolism and cell structure and therefore propose changes in brain structure to be an influential factor in FP, as already described in human neuropsychiatric disorders.

Keywords: Feather pecking, Whole genome sequencing, Imputation, Meta-analysis, Genome wide association study, Chicken

\footnotetext{
* Correspondence: clemens.falker-gieske@uni-goettingen.de

'Department of Animal Sciences, Georg-August-University, Burckhardtweg 2, 37077 Göttingen, Germany

Full list of author information is available at the end of the article
}

(c) The Author(s). 2020 Open Access This article is licensed under a Creative Commons Attribution 4.0 International License, which permits use, sharing, adaptation, distribution and reproduction in any medium or format, as long as you give appropriate credit to the original author(s) and the source, provide a link to the Creative Commons licence, and indicate if changes were made. The images or other third party material in this article are included in the article's Creative Commons licence, unless indicated otherwise in a credit line to the material. If material is not included in the article's Creative Commons licence and your intended use is not permitted by statutory regulation or exceeds the permitted use, you will need to obtain permission directly from the copyright holder. To view a copy of this licence, visit http://creativecommons.org/licenses/by/4.0/. The Creative Commons Public Domain Dedication waiver (http://creativecommons.org/publicdomain/zero/1.0/) applies to the data made available in this article, unless otherwise stated in a credit line to the data. 


\section{Background}

Feather pecking (FP) is a worldwide problem in layers substantially impairing animal welfare and causing economic losses. The propensity to show this behavior is a complex trait affected by various environmental factors and numerous genes. Heritability estimates of around 0.15 were reported $[1-3]$ indicating the possibility to select against this unwanted behavior. Despite decades of intensive research, the causative mechanisms underlying FP are still not completely understood. The mapping of causative genes for FP is challenging because the recording of individual phenotypes requires labor-intensive behavioral observations. Thus, to address this problem, feather condition can be used instead and considered as a social interaction trait affected by FP behavior of conspecifics [4].

It is, however, unpredictable in commercial layer populations, whether and when FP will occur. Thus, most studies have been conducted in layer lines divergently selected for FP behavior [5]. Genomic regions putatively affecting FP were identified by mapping selection signatures within these lines [2, 6], as well as by classical quantitative trait loci (QTL) mapping [7] and genome wide associations study (GWAS) approaches in resource populations established from the lines such as F2 populations [8]. Also, candidate gene approaches [9] have been adopted. In summary, mapping studies identified loci connecting FP to dopaminergic [9], GABAergic [6] and serotonergic signaling $[2,4,9]$ as well as to immune function [4]. Since these studies were based on low-density and mediumdensity marker panels, no candidate mutations were pinpointed. Here we present an approach in which we performed whole-genome sequencing (WGS) on selected animals, in order to impute low-level chip genotype data to sequencing level. We have already successfully applied this strategy in a porcine F2 design [10]. In the present study, we combined the results of GWAS from two resource populations: an F2 design and one half-sib (HS) population divergently selected for feather pecking behavior. This type of meta-analysis was already established in pigs [11] and applied in an FP analysis, which in addition included selection signatures [8]. The same strategy enabled us to identify 15 genome-wide significant variants associated with FP behavior in the present study. The variant with the lowest $p$-value (rs734668878) is an intronvariant of the SPATS2L gene and is located on GGA7. SPATS $2 L$ has been associated with schizophrenia in numerous studies [12-17].

\section{Results}

Whole-genome sequencing, variant calling, and imputation accuracy

An average of 250,771,841 (SD =154,322,025; $\mathrm{MIN}=$ $123,212,910$; $\mathrm{MAX}=815,729,265)$ sequencing reads per sample were aligned to the reference genome with an average mapping efficiency of $98.39 \%$. The mean coverage of sequenced F0 individuals was 14.42 and of HS individuals 23.49. In total 12,864,421 SNPs were detected, $1,219,711$ of which were novel as of dbSNP version Galgal_variation_release-92. With respect to the number of INDELs, $1,561,896$ out of 2,142,539 variants were novel. The Ti/Tv of SNPs was 2.403, whereas known SNPs had a Ti/Tv of 2.449 and novel SNPs had a Ti/Tv of 1.965 . The coefficients of determination for the imputation accuracy with the HS population were as follows: GGA1, $\mathrm{R}^{2}=0.740$ ( $\left.\mathrm{SD}=0.351\right)$, GGA7, $\mathrm{R}^{2}=0.767$ ( $\left.\mathrm{SD}=0.342\right)$. $\mathrm{R}^{2}$ values for all tested markers are summarized in Additional File 1.

\section{Variance explained by markers, GWAS and variant effect prediction}

Based on the SNP-chip data, the phenotypic variance explained by all markers (chip-heritability) was estimated as 0.18 (S.E. 0.05 ) in the F2 and 0.20 (S.E. 0.08) in the HS design, respectively. Using the imputed sequence level data, a GWAS was performed for the trait 'feather pecks delivered' (FPD) in each of the designs. After Bonferroni correction, 16 significant variants ( $p$-value < $7.421 \times 10^{-8}$ ) were detected in the F2 design and 185 significant variants ( $\mathrm{p}$-value $<1.158 \times 10^{-7}$ ) were detected in the HS design. Variants with $-\log 10(\mathrm{p})>5$ are summarized in Additional File 2 and Manhattan plots are shown in Additional File 3. The p-value thresholds after Bonferroni corrections differ due to different numbers of variants analyzed (6,737,823 variants in the F2 design, 4,316,405 variants in the HS structure). Variant effect prediction (VEP) revealed that the two study designs, F2 and HS, have no genes in common among those that are affected by variants with $-\log 10(\mathrm{p})>5$ (Venn diagram in Additional File 4). No statistically significant signals were detected in the two GWAS for the 'posterior probability of animals to be extreme feather peckers' (pEFP) [18] (Manhattan plots in Additional File 3 , variants with $-\log 10(\mathrm{p})>5$ in Additional File 2). The F2 and the HS study designs have no affected genes in common among variants with a $-\log 10(\mathrm{p})>5$ (Venn diagram in Additional File 4).

After performing a meta-analysis of the two FPD data sets, we identified 15 significant variants ( $p$-value $<7.248 \times 10^{-8}, 6,898,216$ variants were included in the analysis), of which 10 variants were also statistically significant in the F2 GWAS and 5 variants in the HS design. A Manhattan plot, which displays the results of the meta-analysis for the phenotype FPD is shown in Fig. 1a and variants with $-\log 10(\mathrm{p})>5$ are summarized in Additional File 2. In the F2 design, the proportion of phenotypic variance jointly explained by all genome-wide significantly associated markers was 
estimated as 0.08 (S.E. 0.04), while the proportion explained by the QTL region in the HS design was estimated as 0.07 (S.E. 0.05). The variants with significant $p$-values are listed in Table 1 . Notably, the meta-analysis of the trait FPD led to the discovery of 111 affected genes, that were not above a $-\log 10(\mathrm{p})$ threshold of 5 in the two single GWAS (Additional File 5). Only one of those genes, UNC50, has been previously associated with FP [19].
No statistically significant variants were found in the meta-analysis of the two pEFP datasets (Fig. 1b), although 16 additional genes were found that are affected by variants with $-\log 10(\mathrm{p})>5$ (Additional File 5 ). The results of the VEP for variants with $-\log 10(\mathrm{p})>5$ for both phenotypes are summarized in Additional File 6.

However, among variants with $-\log 10(\mathrm{p})>5$ the traits FPD and pEFP datasets have 16 affected genes in common (Table 3). It is noteworthy that the variant with the

A

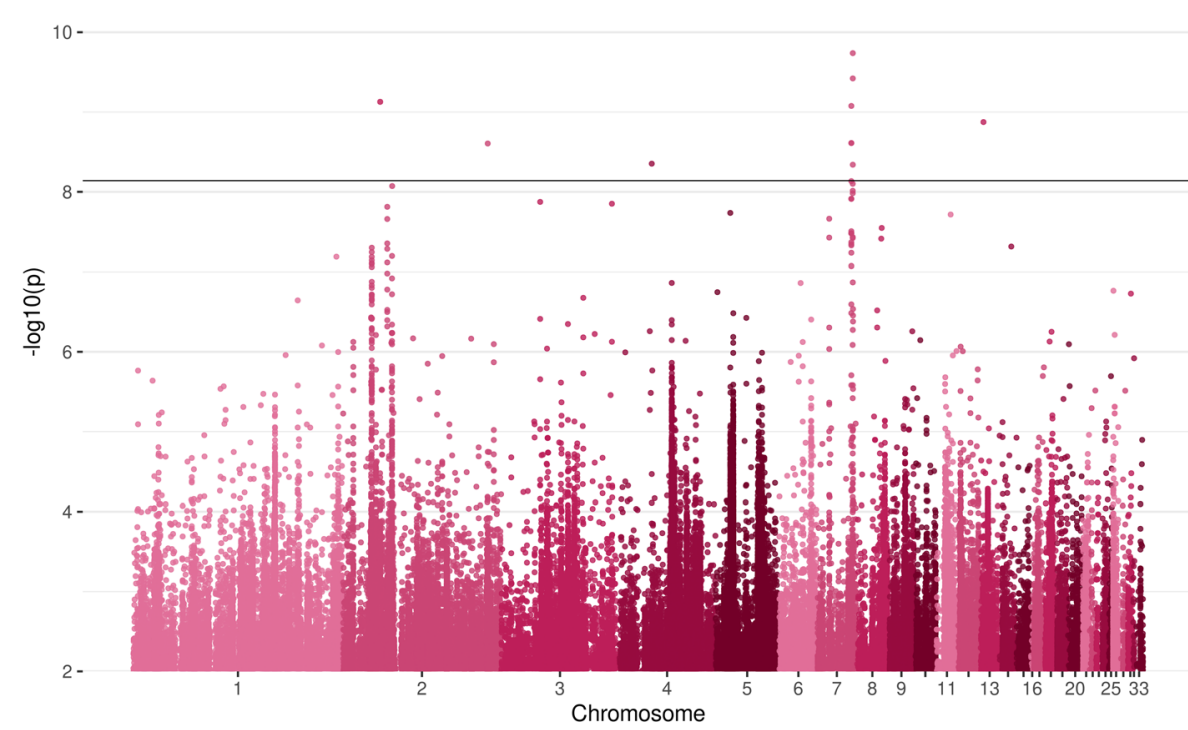

B

$10-$

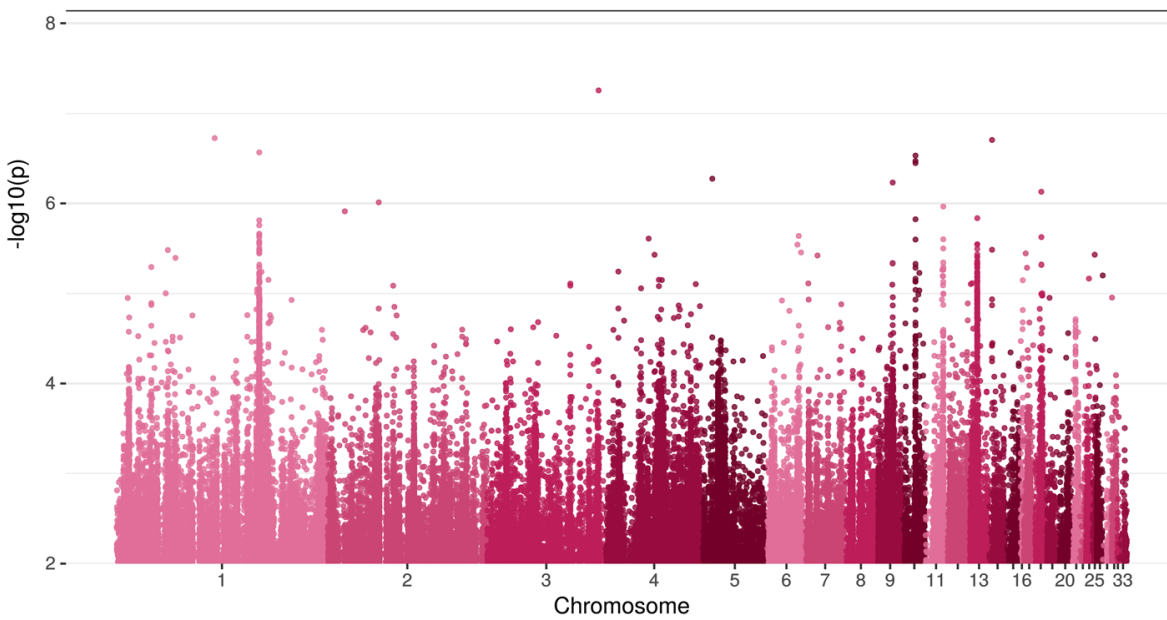

Fig. 1 Manhattan plots of the meta-analyses of genome wide associations studies from two resource populations (F2 and HS; half-sib) with the phenotypes (a) feather pecks delivered' (FPD) and (b) the 'posterior probability of a hen to belong to the extreme feather pecking subgroup' (pEFP) 
Table 1 The variants with significant p-values from the meta-analysis of genome wide associations studies from two resource populations (F2 and HS; half-sib) with the trait 'feather pecks delivered' (FPD)

\begin{tabular}{|c|c|c|c|c|c|}
\hline Chr & Position (bp) & ID & p-value & Variant effect & Symbol \\
\hline 7 & $11,147,641$ & rs734668878 & $3.546 \mathrm{E}-11$ & intron & SPATS2L \\
\hline 7 & $33,398,007$ & rs13600324 & $1.828 \mathrm{E}-10$ & intron & ZEB2 \\
\hline 7 & $33,397,356$ & rs315633354 & $3.791 \mathrm{E}-10$ & intron & ZEB2 \\
\hline 2 & $35,421,866$ & rs794115893 & $7.451 \mathrm{E}-10$ & intergenic & KCNH8 \\
\hline 2 & $35,420,998$ & 2_35,420,998 & 7.451E-10 & intergenic & KCNH8 \\
\hline 2 & $35,422,624$ & rs732446499 & $7.451 \mathrm{E}-10$ & intergenic & KCNH8 \\
\hline 2 & $35,422,366$ & 2_35,422,366 & $7.451 \mathrm{E}-10$ & intergenic & KCNH8 \\
\hline 2 & $35,417,854$ & rs733161790 & $7.451 \mathrm{E}-10$ & intergenic & KCNH8 \\
\hline 7 & $32,004,828$ & rs317095182 & 8.392E-10 & intron & $\angle R P 1 B$ \\
\hline 13 & $3,974,978$ & rs312579842 & $1.336 \mathrm{E}-09$ & intron & FGF18 \\
\hline 7 & $32,009,461$ & rs737373523 & $2.448 \mathrm{E}-09$ & intron & $\angle R P 1 B$ \\
\hline 7 & $32,001,224$ & 7_32,001,224 & $2.448 \mathrm{E}-09$ & intron & $\angle R P 1 B$ \\
\hline 2 & $136,907,571$ & $2 \_136,907,571$ & 2.48E-09 & downstream gene & MRPL13 \\
\hline 4 & $31,165,304$ & rs732945108 & 4.438E-09 & downstream gene & MMAA \\
\hline 7 & $33,397,298$ & rs16611918 & 4.575E-09 & intron & ZEB2 \\
\hline
\end{tabular}

lowest $p$-value for the phenotype pEFP (rs740633021, $p$ value $=5.533 \times 10^{-8}$ ) was also detected in the metaanalysis for FPD ( $\mathrm{p}$-value $\left.=1.410 \times 10^{-8}\right)$. VEP produced two different results for variant rs740633021. It was predicted to be an intron variant of the NRBP1 gene and an upstream gene variant of ncRNA LOC107053158. An

Table 2 The top 20 variants with the lowest $p$-values from the meta-analysis of genome wide associations studies from two resource populations (F2 and HS; half-sib) with the trait 'posterior probability of a hen to belong to the extreme feather pecking subgroup' (pEFP)

\begin{tabular}{|c|c|c|c|c|c|}
\hline Chr & Position (bp) & ID & p-value & Variant effect & Symbol \\
\hline 3 & $104,391,861$ & rs740633021 & $5.533 \mathrm{E}-08$ & intron / upstream gene & NRBP1 / LOC107053158 (ncRNA) \\
\hline 1 & $91,991,270$ & rs737049739 & $1.878 \mathrm{E}-07$ & intergenic & EPHA6 \\
\hline 14 & $2,899,119$ & rs14070749 & $1.968 \mathrm{E}-07$ & non coding transcript / exon & LOC101750426 (ncRNA) \\
\hline 1 & $133,743,444$ & rs15428397 & $2.710 \mathrm{E}-07$ & intron & KIAA1211L \\
\hline 10 & $11,902,712$ & rs317563905 & $2.938 \mathrm{E}-07$ & downstream gene & SH3GL3 / LMINA \\
\hline 10 & $11,904,575$ & rs316617403 & $3.373 \mathrm{E}-07$ & downstream gene / 3 prime UTR & SH3GL3 / LMINA \\
\hline 10 & $11,901,170$ & rs315794181 & $3.564 \mathrm{E}-07$ & downstream gene / 3 prime UTR & SH3GL3 / LMINA \\
\hline 5 & $8,789,444$ & rs312755711 & $5.313 \mathrm{E}-07$ & intron, non coding transcript & LOC107053374 (ncRNA) \\
\hline 9 & $14,663,252$ & rs14674949 & $5.865 \mathrm{E}-07$ & intron & $\angle P P$ \\
\hline 18 & $6,202,927$ & rs736783194 & $7.428 \mathrm{E}-07$ & intergenic & C18H17ORF67 \\
\hline 2 & $48,074,231$ & 2_48,074,231 & $9.747 \mathrm{E}-07$ & intron & PDEIC \\
\hline 11 & $16,848,616$ & rs732938021 & 0.000001084 & intron & KLHL36 \\
\hline 2 & $16,335,664$ & rs731673476 & 0.000001226 & intron & MYOЗА \\
\hline 13 & $8,132,912$ & rs739578589 & 0.000001461 & intron & GABRB2 \\
\hline 10 & $11,904,145$ & rs313724095 & 0.000001503 & downstream gene / 3 prime UTR & SH3GL3 / LMINA \\
\hline 1 & $133,734,332$ & rs14889804 & 0.000001544 & intron & KIAA1211L \\
\hline 1 & $133,762,004$ & rs13624872 & 0.000001753 & intron & KIAA1211L \\
\hline 1 & $133,776,463$ & rs313923430 & 0.000002173 & intron & TSGA10 \\
\hline 1 & $133,733,974$ & rs1059691759 & 0.000002254 & intron & KIAA1211L \\
\hline 6 & $29,989,563$ & $6 \_29,989,563$ & 0.000002303 & intron & SHTN1 \\
\hline
\end{tabular}


intron variant affecting SPATS2L (rs734668878, FPD $p$ value $=3.546 \times 10^{-11}, \mathrm{pEFP}$-value $=3.804 \times 10^{-6}$ ) was detected in the meta-analyses of both phenotypes as well.

Positive genetic correlations between FP and performance have been reported [20], which might be regarded as a cause for the frequent occurrence of FP in layers. Thus, we also compared our gene lists (Tables 1,2 and 3) with gene entries in the ChickenQTLdb (https://www.animalgenome.org/cgi-bin/QTLdb/GG/ index) related to QTL for production traits and only identified a single gene. $L P P$, noteworthy one of the genes found in common between FPD and pEFP (Table 3 ), has been reported to be linked to shank length and diameter [21] as well as Bursa of Fabricius weight [22]. The latter finding might correspond the observation of feather pecking being related to the immune system, but this is speculative and needs to be further investigated.

\section{Functional and pathway analyses}

Gene cluster analysis with the R package clusterProfiler [23] was performed with genes affected by variants with $-\log 10(\mathrm{p})>5$ from the meta-analyses of GWAS performed on the phenotypes FPD and pEFP. Significant results (q-value $>0.25$ ) that lead to the discovery of categories affected by more than one gene are shown in Table 4. Since the analysis of GO cellular components for the trait FPD led to the discovery of more than 20 terms the results are shown in Fig. 2. The complete clusterProfiler output is summarized in Additional File 7.

Protein interaction network analysis of genes affected by variants with $-\log 10(\mathrm{p})>5$ associated with FPD in the meta-analysis with STRING revealed significantly more interactions than expected (number of edges: 54, expected number of edges: 40, PPI enrichment $p$-value $=$ 0.0197, Fig. 3). Several protein clusters with more than 3 nodes were found: cluster (i) ATP8A2, STIM2, ITPR1, PLCG2, DGKB, DGKE, GPD2, LRP1B, FGGY, and LOC429153; cluster (ii) ECT2, DIAPH3, DIAPH1, TAOK1, and PARD3B; cluster (iii) MLH1, IGF2, VTI1B, and ISLR; cluster (iv) SAMD1, ZEB2, ZFHX3, and GTDC1; cluster (v) HACE1, HERC4, LMO7, and LPP; cluster (vi) EXOC7, DAB2IP, LXN, and TP63. Analysis of the two resource populations separately yielded significantly more interactions than expected in the HS design (number of edges: 17, expected number of edges: 8 , PPI enrichment $p$-value $=0.00511$ ) but not in the F2 cross (Additional File 8). The same holds true for the pEFP phenotype.

\section{Discussion}

In the study presented here, we imputed mediumdensity SNP-chip genotypes of two layer lines divergently selected for FP to sequence level. GWAS and subsequent meta-analyses for the traits 'feather pecks delivered' (FPD) and 'posterior probability of belonging to the subgroup of extreme feather peckers' ( $\mathrm{pEFP}$ ) were performed with both populations. We detected 16 variants

Table 3 Common genes from the genome wide associations studies meta-analyses that are affected by variants with a -log $10(p)>5$ for the phenotypes 'feather pecks delivered' (FPD) and the 'posterior probability of a hen to belong to the extreme feather pecking subgroup' (pEFP)

\begin{tabular}{|c|c|c|c|c|}
\hline Symbol & ID (FPD) & p-value (FPD) & ID (pEFP) & $p$-value (pEFP) \\
\hline COIL & rs739114475 & $5.613 \mathrm{E}-07$ & rs731484806 & 4.79E-06 \\
\hline DGKE & rs739114475 & 5.613E-07 & rs731484806 & 4.79E-06 \\
\hline KIAA1211L & rs314041779 & 0.000003441 & rs15428397 & $2.71 \mathrm{E}-07$ \\
\hline LMINA & rs13544839 & 0.000006722 & rs317563905 & $2.938 \mathrm{E}-07$ \\
\hline LOC101750426 & rs312554616 & 0.000007613 & rs14070749 & $1.968 \mathrm{E}-07$ \\
\hline LOC101752088 & 18_7107365 & 0.000006267 & rs794664310 & 0.000009907 \\
\hline LOC107053158 & rs740633021 & $1.41 \mathrm{E}-08$ & rs740633021 & 5.533E-08 \\
\hline$\angle P P$ & rs314190948 & 0.000009257 & rs14674949 & 5.865E-07 \\
\hline NRBP1 & rs740633021 & $1.41 \mathrm{E}-08$ & rs740633021 & 5.533E-08 \\
\hline PITPNC1 & 18_7107365 & 0.000006267 & rs794664310 & 0.000009907 \\
\hline RUFY3 & rs732635903 & $1.371 \mathrm{E}-07$ & 4_50027189 & 0.000007014 \\
\hline SCPEP1 & rs739114475 & $5.613 \mathrm{E}-07$ & rs731484806 & 0.000004791 \\
\hline SH3GL3 & rs13544839 & 0.000006722 & rs317563905 & 2.938E-07 \\
\hline SPATS2L & rs734668878 & $3.546 \mathrm{E}-11$ & rs7346688878 & 0.000003804 \\
\hline TSGA10 & rs738236556 & 0.000004947 & rs313923430 & 0.000002173 \\
\hline UTP3 & 4_50027189 & 4.013E-07 & 4_50027189 & 0.000007014 \\
\hline
\end{tabular}


Table 4 Gene set enrichment analysis of genes affected by variants $(-\log 10(p)>5)$ from the genome wide associations studies meta-analyses with the traits 'feather pecks delivered' (FPD) and the 'posterior probability of a hen to belong to the extreme feather pecking subgroup' (pEFP) with clusterProfiler. Results with q-values $>0.25$ and at least two gene symbols are listed

\begin{tabular}{llllll}
\hline Category & ID & Description & q-value & Symbols & Phenotype \\
\hline KEGG & gga04070 & Phosphatidylinositol signaling system & 0.000156805 & MTMR7/INPP4B/PLCG2/DGKB/DGKE/TPR1 & FPD \\
GO_BP & GO:0006897 & endocytosis & 0.230358006 & CDH13/SH3GL3 & pEFP \\
GO_BP & GO:0098657 & import into cell & 0.230358006 & CDH13/SH3GL3 & pEFP \\
GO_CC & GO:0005912 & adherens junction & 0.053757065 & CDH13/LPP & pEFP \\
GO_CC & GO:0070161 & anchoring junction & 0.053757065 & CDH13/LPP & pEFP \\
GO_CC & GO:0098805 & whole membrane & 0.098234855 & CDH13/SH3GL3 & pEFP \\
GO_CC & GO:0030054 & cell junction & 0.098234855 & CDH13/LPP & PEFP \\
\hline
\end{tabular}

that are significantly associated with the trait FPD. The variant with the lowest $p$-value (rs734668878, $p$-value $=$ $3.546 \times 10^{-11}$ ) is an intron-variant in the SPATS2L gene. Polymorphisms of SPATS2L have been linked to hippocampal volume, intelligence, and schizophrenia [12-17], which makes it a promising candidate gene to have an influence on FP behavior. Three statistical significant variants are located in intronic regions of ZEB2 (lowest $p$-value $\left.1.828 \times 10^{-10}\right)$, a gene which has been identified to be a risk locus for schizophrenia [24] and to be inducible by nicotine [25]. In proximity to $K C H N 8$ we discovered five significant variants (lowest p-value $7.451 \times$ $10^{-10}$ ). KCNH8 was identified in the context of the neuropsychiatric $15 \mathrm{q} 13.3$ microdeletion which is associated with several neuropsychiatric disorders including autism, schizophrenia, and attention deficit hyperactivity

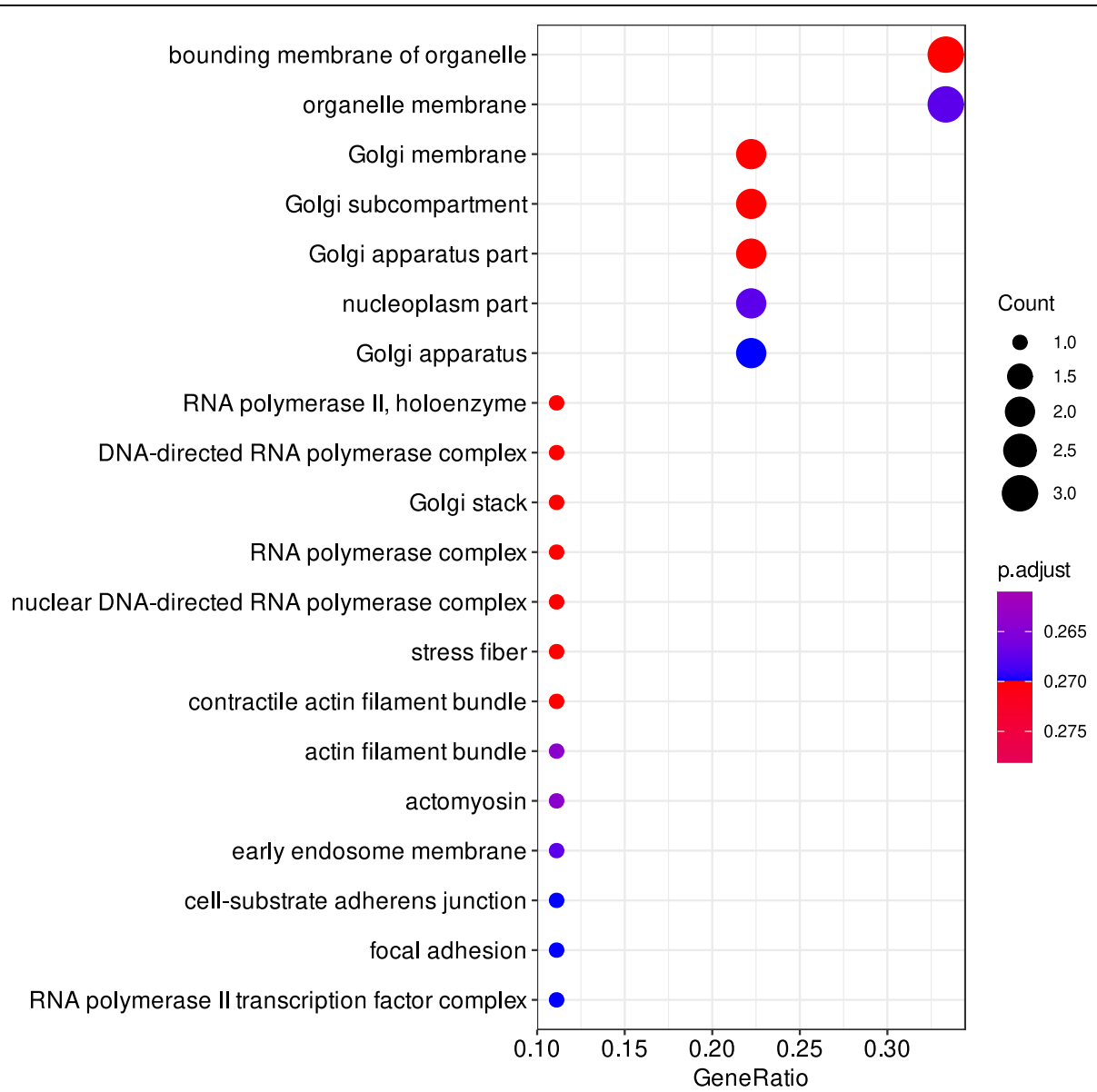

Fig. 2 Enriched GO cellular components terms for genes that contain variants with $-\log 10(p)>5$ that were discovered in the meta-analysis of two genome wide associations studies for the trait 'feather pecks delivered' (FPD) 


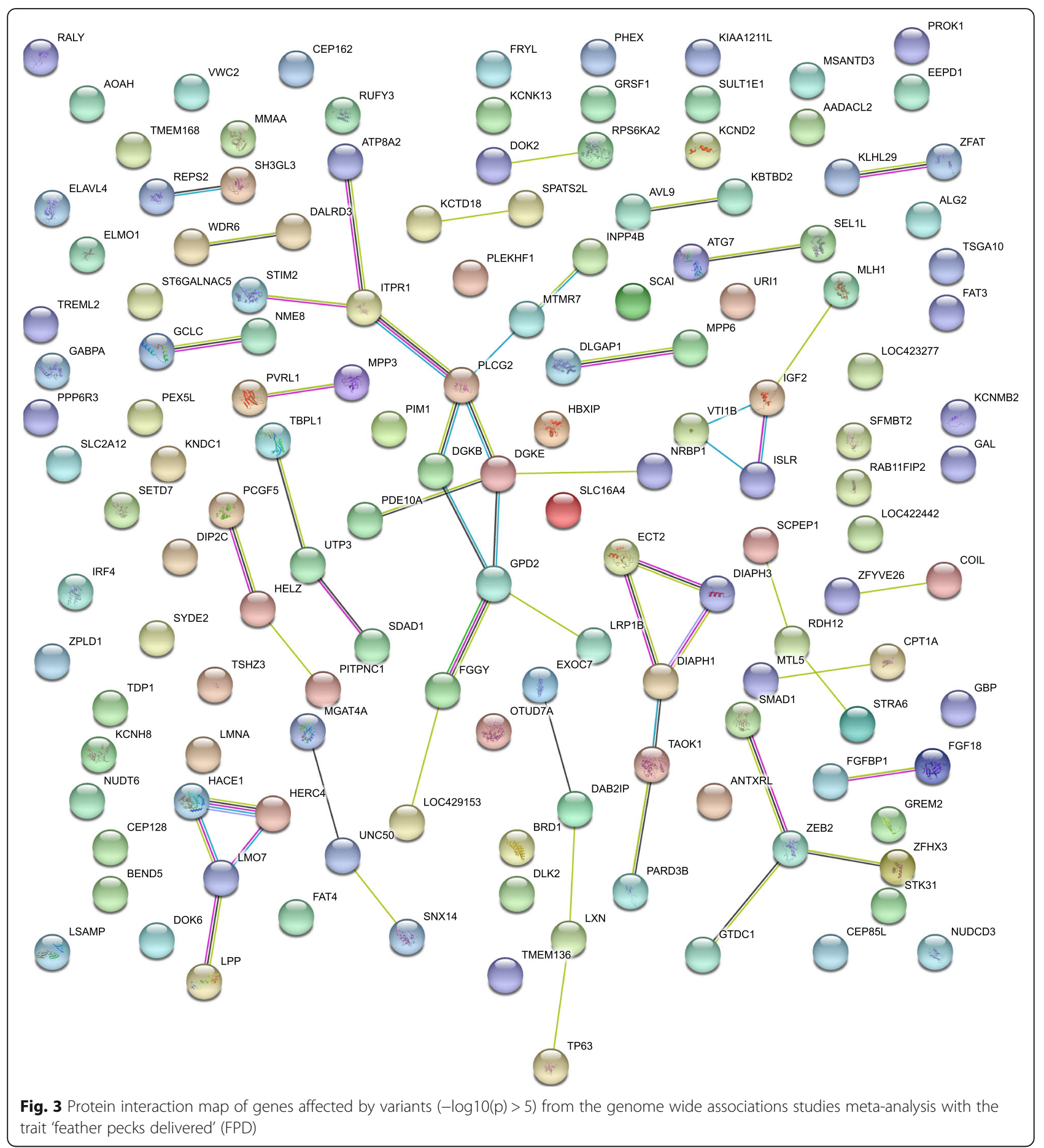

disorder [26]. Furthermore, we found three intronvariants in $L R P 1 B$ (lowest $p$-value $8.392 \times 10^{-10}$ ) to be significantly associated with the trait FPD. $L R P 1 B$ was identified in a GWAS where it was found to be associated with increased ventricular volumes in psychosis [27]. Another promising gene that might influence the propensity to FP is FGF18. We found a significantly associated intron-variant (rs312579842, p-value $1.336 \times$
$10^{-9}$ ) in FGF18, a gene that has been connected to dyslexia [28]. Furthermore, dysregulation of fibroblast growth factor signaling is currently under discussion to play a role in neurological and psychiatric disorders [29]. Another gene affected by a significantly associated down-stream gene variant is MRPL13 (2_136,907,571, pvalue $=2.480 \times 10^{-9}$ ), which was found to be upregulated after nicotine treatment [30] like the already mentioned 
ZEB2 gene. We found genes encoding subunits of nicotinic acetylcholine receptors (nAchR), the major receptors for nicotine [31], to be differentially expressed in the brain transcriptome of hens divergently selected for feather pecking [32]. Both findings taken together present strong evidence for the involvement of nAchR in the propensity to FP behavior (already discussed in [32]). Gene cluster analysis with clusterProfiler revealed that genes belonging to the KEGG category "Phosphatidylinositol signaling system" are enriched among genes associated with the trait FPD (Table 4). This discovery is of special interest since the phosphatidylinositol second messenger system is currently under discussion to be involved in the pathophysiology of psychiatric disorders [33]. Furthermore, GO cellular component analysis (Fig. 2) revealed that genes located in the Golgi apparatus and genes involved in cell structure are affected by variants identified in this study. Golgipathies and Golgi fragmentation are currently a matter of discussion for an influential role in neurodegenerative diseases and neurological development [34, 35].

In summary, the polymorphisms affecting the genes discussed above substantially contribute to the explanation of the genetic architecture behind FP behavior. While we estimated a chip heritability that corresponds well with previously published genetic parameters [1-3], the associated variants in this study do explain $7-8 \%$ of phenotypic variance for FPD.

Since we discovered more protein interactions among FPD-associated gene products than expected (Fig. 3), we propose that this interaction map provides a basis for downstream functional analysis. The proteins that belong to the largest cluster (cluster (i): ATP8A2, STIM2, ITPR1, PLCG2, DGKB, DGKE, GPD2, LRP1B, FGGY, and LOC429153) confirm the finding from our gene cluster analysis. A large number of these proteins are involved in phosphatidylinositol metabolism but also in other lipid- and membrane-associated processes. The second-largest cluster contains the proteins ECT2, DIAP H3, DIAPH1, TAOK1, and PARD3B, all of which play a role in structural processes. We found another cluster that contains genes, which influence brain development and cell proliferation in the brain (cluster (iv) SAMD1, ZEB2, ZFHX3, and GTDC1). This confirms our findings from the GO cellular component analysis (Fig. 2). Structural anomalies of the brain have been shown to be involved in numerous psychiatric disorders [36]. A comparative analysis of the brain structure of hens that exhibit FP behavior with control animals is necessary to clarify, whether this also holds true for the FP phenotype. Another protein interaction cluster consists of MLH1, VTI1B, ISLR, and IGF2. The IGF2 gene is especially interesting since it is currently under discussion as a pharmacological target in various human psychiatric conditions (reviewed in [37]). Interaction cluster (v) contains proteins that are involved in ubiquitin transfer (HACE1, HERC4, LMO7, and LPP). The ubiquitin proteasome system is disrupted in neuropsychiatric disorders and has important functions in brain development [38].

In a second meta-analysis, the pEFP as described by Iffland et al. [18] was examined. Extreme feather pecking (EFP) hens are a subgroup of hens that exhibit FP behavior and in case of the F2 cross more than twice as often as hens of the normal feather pecking subgroup. They peck with higher intensities, and peck up to five times more feathers. Interestingly, EFP hens can be found in lines selected for high (HFP) and low feather pecking (LFP) and make up about one-third of the HS population as well as the F2 cross $[6,18]$. It seems that extreme pecking and overall propensity to show FP behavior are phenotypically highly correlated, but clearly not identical traits. Thus, overlaps between GWAS results for pEFP and FPD cannot necessarily be expected. Here, significant signals for pEFP were neither detectable in the GWAS of the two resource populations (Additional File 3) nor in the meta-analysis after Bonferroni correction (Fig. 1b). However, we were able to correlate our findings with previous studies by examining variants with $-\log 10(\mathrm{p})>5$. In a previous study partly using the same resource populations, a significant QTL on GGA1 (131, 055,669 - 133,345,452 bp) was identified, which was confirmed in the current meta-analysis (Fig. 1b, 131,766,790 - 134,135,880 bp). Based on their mapping results, Iffland et al. [6] reported different GABA-receptor subunits (GABRA5, GABRB3, and GABRG3) as potential candidate genes. That study was carried out using SNPchip data providing limited mapping resolution. Inspecting the genes in our meta-analysis, using VEP revealed that NIPA1, KIAA1211L, AFF3, and TSGA10 are genes affected by variants in this region. Copy number variations in NIPA1 were found in psychiatric disorders [39], KIAA1211L was identified in a schizophrenia twin-study [40] and is a candidate gene for opioid abuse [41], and $A F F 3$ is involved in intellectual disability and cellular migration in the cerebral cortex of mice [42]. The studies mentioned make those genes likely candidates to influence the trait pEFP. However, we did not find evidence for the involvement of the GABA-receptor subunits found by Iffland et al. [6]. In the previous study, medium-density SNP chip data was used and candidate genes were thus identified based on linkage disequilibrium. Our current approach using imputed sequence level genotypes proves superior in this respect as the evaluation of variants within or close to putative candidate genes is possible. Although we were not able to confirm the aforementioned genes related to GABAergic signaling, we were able to detect 52 intron variants, 
among them two insertions, with $-\log 10(\mathrm{p})>5$ on GGA13 (8,084,380 - 8,135,739bp), which affect the GABA-receptor subunit GABRB2 (Additional File 2). For $G A B R B 2$ as well strong evidence exists to be a causative gene for schizophrenia [43-50] and it seems to be involved in opioid addiction [51]. Iffland et al. [6] found the QTL region on GGA1 also associated with FPD, but we were not able to confirm this in our GWAS based on imputed sequence level genotypes. Nevertheless, we found 16 common genes out of 40 (pEFP) and 191 (FPD) affected by polymorphisms with $-\log 10(\mathrm{p})>5$ between the two traits (Table 3 ) by which we also confirm a small overlap.

Imputation accuracy in the HS population was considerably high with average coefficients of determination between $R^{2}=0.740-0.767$. We assume that the imputation accuracy in the F2 population was even higher, due to the usage of pedigree information. However, because of the lack of sequenced individuals we were not able to compute it. Notably, the GWAS for both traits revealed no common significantly associated genes $(-\log 10(\mathrm{p})>5)$ between the F2 and HS populations (Venn diagrams in Additional File 4 and gene lists in Additional File 5). It seems possible that the top GWAS hits in the F2 population are based on loci that are fixed or close to fixation in the pure selection lines and are thus not detectable in the HS structure. On the other hand, loci still segregating in one of the lines will be easily detectable if sires are QTL heterozygous.

\section{Conclusions}

We identified several significant genomic variants associated with the trait FPD, that mostly affect genes that have been previously associated with psychiatric and neurological disorders. Among them SPATS $2 L$, $Z E B 2$, and $K C H N 8$, all of which have been identified in schizophrenia studies [12-17, 24, 26]. Furthermore, the genes $Z E B 2$ and $M R P L 13$, which are affected by significantly associated variants, respond to nicotine treatment $[25,30]$. This goes in line with our previous results where we detected differential expression of major receptors for nicotine between HFP and LFP hens [32]. Based on our findings from gene cluster analysis and protein interaction clustering we also propose an involvement of phosphatidylinositol signaling, disturbances of the Golgi apparatus, and structural abnormalities in the brain as possible factors that influence the predisposition for FP behavior. Concerning the trait pEFP we could confirm a QTL on GGA1 that was previously identified [6], which in our study contains variants affecting NIPA1, $K I A A 1211 L$, AFF3, and TSGA10. All of those genes, except TSGA10, have been implicated in neurological conditions [39-42] making them additional targets for genomic selection against FP.

\section{Methods \\ Experimental population}

Both experimental populations were derived from White Leghorn lines divergently selected for low and high feather pecking $[2,52]$. These lines were created and are maintained at the Hohenheim University and neither commercially obtained nor from a private source. The F2-design was established from the 10th generation of the selection experiment and has been described in detail by Lutz et al. [8]. Briefly, five sires and ten dams of each line were used to generate $10 \mathrm{~F} 1$ families; then, 10 F1 sires were used to generate the F2 families. Each sire was mated with eight F1 hens four times by artificial insemination. In total, 960 F2 offspring were produced in four hatches. The half-sib design (HS) was recruited from the 15th generation of the selection experiment by mating six sires per line to hens within the respective line with the aim to produce about 600 animals in total. Animals were phenotyped according to established protocols (see below) and genotyped using an Illumina $60 \mathrm{k}$ SNP chip. We successfully pheno- and genotyped a total of 817 F2 hens and 489 offspring from the HS structure (270 HFP and 219 LFP animals). Chickens were $\mathrm{CO}_{2}-$ stunned and sacrificed by ventral neck cutting. Blood was drawn directly while the animals were bleeding out.

\section{Phenotyping}

Details on phenotyping and housing conditions can be found in Lutz et al. [8] and Iffland et al. [6]. Briefly, animals were phenotyped at around 32 weeks of age according to established protocols $[53,54]$ recording the feather pecks delivered. For the pure lines, HFP and LFP animals were kept in a ratio as close to $1: 1$ as possible. The group size was around 42 animals. Observations were done in sessions of $20 \mathrm{~min}$ by seven independent and trained observers. Phenotypic values were standardized to $420 \mathrm{~min}$ observation time (referred to as FPD in the manuscript). In addition, we derived the posterior probability of belonging to the subgroup of extreme feather peckers (pEFP) as previously described [18, 55]. This is briefly achieved by fitting a mixture of two negative binomial distributions to FP data $[6,18]$.

\section{DNA isolation}

DNA was isolated from EDTA conserved blood samples with the Promega Maxwell $^{\oplus} 16$ and Maxwell ${ }^{\odot}$ Blood DNA Purification Kits (Kat\# AS1010) according to the manufacturer's protocol. For genotyping with Illumina SNP chips the concentration was adjusted to $50+/-10$ $\mathrm{ng} / \mu \mathrm{l}$. 


\section{NGS library preparation and sequencing}

Illumina PCR-free TruSeq fragment libraries with $350 \mathrm{bp}$ insert size were prepared. We collected on average 128 million $2 \times 150$ bp paired-end reads on a NovSeq6000 instrument.

\section{Mapping and variant detection}

Mapping and variant calling was performed according to the GATK best practice pipeline using GATK v. 4.0 [56] and chicken genome version GRCg6a (GCF 000002315.5 RefSeq assembly). Briefly, fastq files were converted to bam files with GATK FastqToSam in order to add read group information (Instrument Name, Library ID, run ID, flowcell ID, and flowcell lane). Illumina adapters were marked with GATK MarkIlluminaAdapters. Reads were mapped to the reference genome with bwa-mem version 0.7.12 [57]. Technical artifacts and duplicate reads were marked with GATK MarkDuplicates. Base quality scores were recalibrated with GATK BaseRecalibrator and ApplyBQSR. Variants were called by sample with GATK HaplotypeCaller in ERC mode. The resulting GVCF files were merged with GATK CombineGVCFs and jointly genotyped with GATK GenotypeGVCfs. The resulting raw variants were filtered with GATK VariantRecalibrator and ApplyVQSR. The knownSites dataset was produced by performing a liftover of Galgal_variation_release-92 (ensemble) from genome Version Gallus_gallus-5.0 (ensemble) to GRCg6a with GATK LiftoverVcf. The chain file for the lift-over was created with flo (https://github.com/wurmlab/flo) with both genomes as input files. The success rate of the dbSNP liftover was $99.35 \%$. Base quality score recalibration was performed with the aforementioned dbSNP version as the knownSites dataset. Truth datasets used for Variant Quality Score Recalibration (VQSR) were as follows. SNPs: Illumina chicken $60 \mathrm{k}$ BeadChip and $600 \mathrm{k}$ Affymetrix Axiom HD chicken genotyping array. INDELs: High confidence fraction (filter settings: QD 30.0, FS 200.0, ReadPosRankSum 20.0) of a variant calling performed on a dataset of 50 laying hens (25 brown layers and 25 white layers) that were already described [58]. Training dataset for SNP VQSR was also a high confidence fraction of the dataset by $\mathrm{Ni}$ et al. (filter settings: QD 30.0, FS 60.0, MQ 40.0, MQRankSum 12.5, ReadPosRankSum 8.0, SOR 3.0). A truth sensitivity of 99.0 was chosen for SNPs and INDELs. The known dataset for SNP and INDEL VQSR was the dbSNP database mentioned above. Since SNPs were filtered with two truth datasets, a Ti/Tv free recalibration according to the GATK best practice guidelines was applied to the data. Variant IDs for novel variants were generated automatically by combining the chromosome number with the variant position in bp.

\section{Haplotype construction and imputation}

Haplotype phasing and imputation of the various datasets was performed with Beagle 5.0. Beagle 4.0 was used when pedigree data was involved, since it is the latest version to support pedigree data. The phasing/imputation workflow is summarized in Fig. 4. Prior to imputation, SNP chip data was filtered excluding monomorphic and unplaced markers as well as markers on sex chromosomes and with a call rate of less than 95\%. Variants from WGS data were called jointly and used as a reference population for the imputation of the HS chip data and the F1 chip data. Imputed F1 genotypes were merged with the WGS genotype data and used as a reference population for the imputation of the F2 chip level genotypes. To compute the imputation accuracy imputation of the HS population was repeated 4 times, leaving out 4 animals that were chip genotyped and sequenced. The imputed genotypes for each animal were extracted from the files and merged. The WGS genotypes of the same animals were extracted from the variant call file and correlation (coefficient of determination, $\mathrm{R}^{2}$ ) for each variant on GGA1 and GGA7 was calculated with an in house R script. In total, 24 of the HS animals were sequenced for the reference population. None of the F1 and F2 animals were sequenced.

\section{Genome wide association studies}

GWAS were performed with the Genome-wide Complex Trait Analysis (GCTA) software version 1.92 .3 beta3 using the imputed sequence level genotype data filtered for MAF (>0.01). In order to correct for relatedness between animals and stratification [6], a random genetic term was included based on a genomic relationship matrix calculated only from SNP-chip-data and following a leave one chromosome out (LOCO) approach as described here [10]. Briefly, a model of the form $y=$ $\boldsymbol{W} \boldsymbol{\alpha}+\boldsymbol{X} \boldsymbol{\beta}+\boldsymbol{u}+\boldsymbol{\epsilon}$ is fitted, where $\mathbf{y}$ is an $\mathbf{n} \times \mathbf{1}$ vector of phenotypes $\mathbf{n}$ hens (a summary of the phenotype data is shown in Table 5). $\mathbf{W}$ is an $\mathbf{n} \times \mathbf{c}$ incidence matrix of fixed effects with $\mathbf{c}$ being the number of effects and $\boldsymbol{\alpha}$ is a vector of corresponding coefficients including the mean. Further, the effects of line (HFP and LFP) and hatch were included in the analysis of the HS design, while only hatch was included for the F2 design. $\mathbf{X}$ is an $\mathbf{n} \times \mathbf{1}$ vector of marker genotypes at the locus tested and $\boldsymbol{\beta}$ is the corresponding effect size; and $\mathbf{u}$ is a vector of random genetic effects, with $\boldsymbol{u} \sim N\left(0, \boldsymbol{A}^{-} \sigma_{g}^{2}\right)$, where $\sigma_{g}^{2}$ represents genetic variance and $\mathrm{A}^{-}$is the genomic relationship matrix based on all SNP-chip markers except those on the chromosome currently analyzed. Finally, $\boldsymbol{\varepsilon}$ is a random residual term, with $\boldsymbol{\epsilon} \sim N\left(0, \boldsymbol{I} \sigma_{\epsilon}^{2}\right)$, where $\sigma_{\epsilon}^{2}$ represents the residual variance and $\mathbf{I}$ represents an identity matrix. 


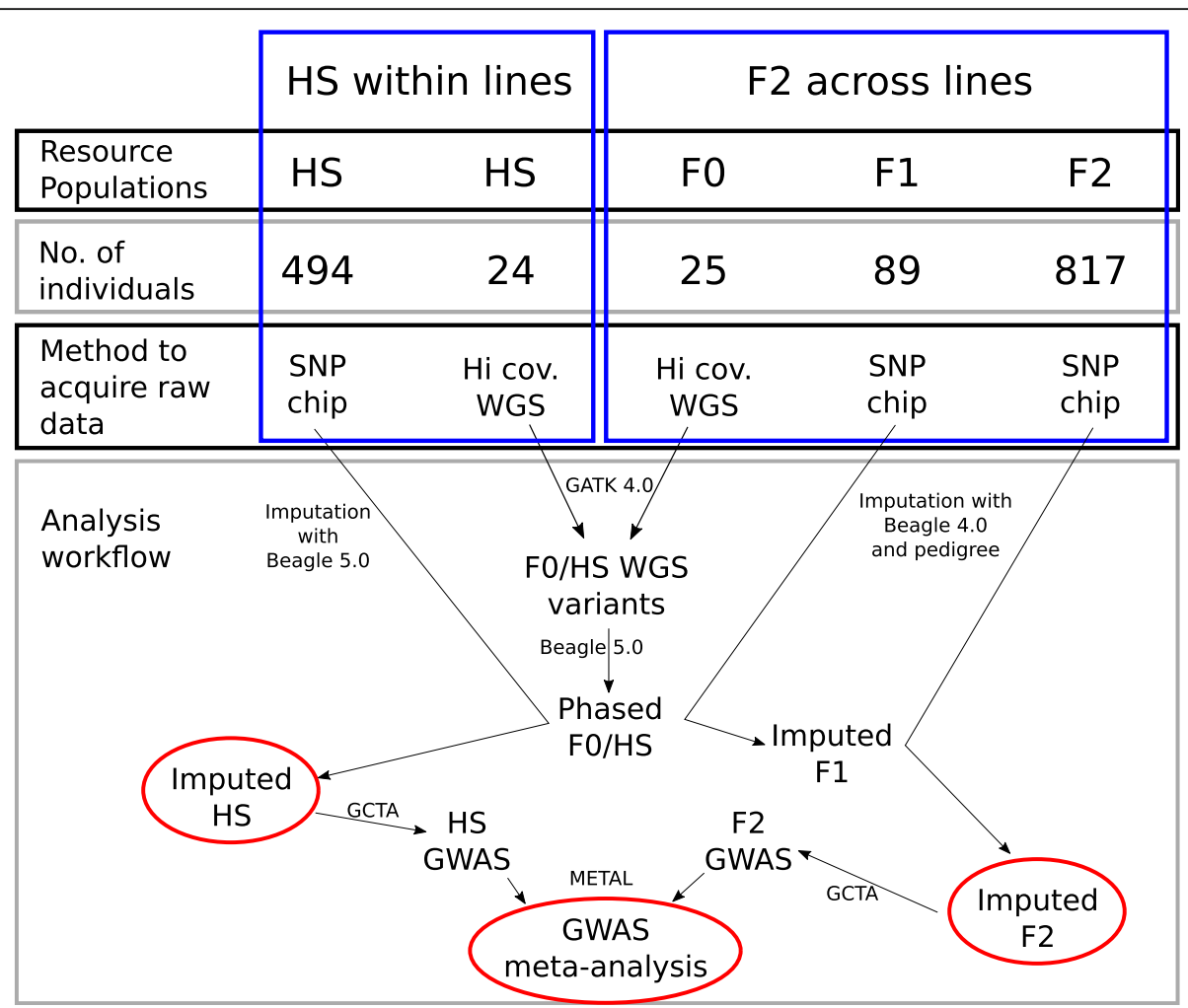

Fig. 4 Analysis workflow from raw data to genome wide associations studies meta-analysis results. High coverage whole-genome sequencing (WGS) variants from an F2-cross and a half-sib (HS) population were used as a reference population for the imputation of low-density single nucleotide polymorphism (SNP) chip genotypes

The variance explained by all markers on the SNPchip was estimated using the same model without the SNP effect. In the F2 design, the variance explained by all significantly associated markers was estimated by including a second GRM based on the significant SNPs. As in the HS design only a single significant QTL was found with several adjacent markers, we partitioned the variance into a component explained by the SNP chip

Table 5 Summary of phenotype values used in the genome wide analysis studies for the traits 'feather pecks delivered' (FPD) and the 'posterior probability of a hen to belong to the extreme feather pecking subgroup' (pEFP)

\begin{tabular}{llll}
\hline & F2 & HS HFP & HS LFP \\
\hline No. of animals & 817 & 270 & 221 \\
FPD average & 13.91 & 10.35 & 1.50 \\
FPD SD & 26.19 & 16.69 & 1.94 \\
FPD min & 0 & 0 & 0 \\
FPD max & 198.33 & 115.50 & 15 \\
pEFP average & 0.28 & 0.31 & 0.20 \\
PEFP SD & 0.45 & 0.29 & 0.15 \\
pEFP min & 0 & 0.12 & 0.11 \\
pEFP max & 1 & 1 & 1 \\
\hline
\end{tabular}

markers within a $5 \mathrm{Mb}$ QTL window and the rest of the genome.

Meta-analysis of the GCTA output for each trait was performed with METAL version 1.1 using the sample size based approach with default settings [59].

\section{Variant effect prediction}

The Ensembl Variant Effect Predictor release 98, which is part of the Ensembl advanced programming interface (API), was used for variant effect prediction with the following settings: --cache --species chicken --refseq --force_overwrite --variant_class --symbol --nearest symbol --sift b --tab.

\section{Functional analyses}

Gene cluster analyses were conducted using the R package clusterProfiler [23] along with the chicken genome annotation org. Gg.eg.db [60] and using default settings in terms of gene set size and $p$-value correction. Protein interaction networks were computed with STRING v. 11 [61] with default settings.

\section{Supplementary information}

Supplementary information accompanies this paper at https://doi.org/10. 1186/s12863-020-00920-9. 
Additional file 1. Coefficients of determination of imputation accuracy on GGA1 and GGA7 for the HS population.

Additional file 2. Variants $(-\log 10(p)>5)$ from all GWAS and metaanalysis performed with the traits FPD and pEFP.

Additional file 3. Manhattan plots of the GWAS performed with the F2 cross and the HS structure with the traits FPD and pEFP.

Additional file 4. Venn diagrams of associated genes from the GWAS and meta-analyses with the traits FPD and pEFP.

Additional file 5. List of genes concordant and discordant between the GWAS and meta-analyses with the traits FPD and pEFP.

Additional file 6. Results of the VEP of genes affected by variants $(-\log 10(p)>5)$ from the GWAS and meta-analyses with the traits FPD and pEFP.

Additional file 7. Results of the gene set enrichment analysis of genes affected by variants $(-\log 10(p)>5)$ from the GWAS meta-analyses with the traits FPD and pEFP with clusterProfiler.

Additional file 8. Protein interactions maps of genes affected by variants $(-\log 10(p)>5)$ from the GWAS with the traits FPD and pEFP.

\section{Abbreviations}

EFP: Extreme feather pecking; FP: Feather pecking; FPD: Feather pecks delivered; FPKM: Fragments per kilobase of exon model per million reads mapped; GWAS: Genome wide associations study/studies; HFP: High feather peckers; HS: Half-sib; LFP: Low feather peckers; nAchR: nicotinic acetylcholine receptors; ncRNA: non-coding RNA; NMDARs: N-methyl-d-aspartate receptors; pEFP: posterior probability of hens to belong to the extreme feather pecking subgroup; VEP: Variant effect prediction; WGS: Whole genome sequencing

\section{Acknowledgements}

We thank Andrea Mott, Hannah Hellmold and Judith Beier for sample collection and phenotyping and the teams at the Institute of Animal Science and the Research station "Unterer Lindenhof" for expert technical assistance. We further acknowledge support by the Open Access Publication Funds of the Göttingen University.

\section{Authors' contributions}

CFG performed all bioinformatics analyses and wrote the manuscript. $\mathrm{HI}$ participated in sample collection and phenotyping and analyzed phenotypic data. SP participated in sample collection and DNA extraction from blood. CD performed the sequencing. WB and JB developed the project outline. JT developed the project outline, developed data analysis strategies, and wrote the manuscript. All authors have read and approved the manuscript.

\section{Funding}

The study was funded by the German Research Foundation (DFG) under file numbers TE622/4-2 and BE3703/8-2. The funding bodies did not contribute to the design of the study or collection, analysis and interpretation of data and writing the manuscript. Open Access funding enabled and organized by Projekt DEAL.

\section{Availability of data and materials}

The datasets used and/or analysed during the current study are available from the corresponding author on reasonable request. The raw WGS data is available under BioProject ID PRJNA664592.

\section{Ethics approval and consent to participate}

The research protocol was approved by the German Ethical Commission of Animal Welfare of the Provincial Government of Baden-Wuerttemberg, Germany (code: $\mathrm{HOH}$ 35/15 PG, date of approval: 25 April 2017).

\section{Consent for publication}

Not applicable.

\section{Competing interests}

Jens Tetens is associate Editor of BMC Genetics.

\section{Author details}

Department of Animal Sciences, Georg-August-University, Burckhardtweg 2, 37077 Göttingen, Germany. ${ }^{2}$ Institute of Animal Science, University of Hohenheim, Garbenstr. 17, 70599 Stuttgart, Germany. ${ }^{3}$ Institute of Genetics, Vetsuisse Faculty, University of Bern, Bremgartenstr. 109a, 3001 Bern, Switzerland. ${ }^{4}$ Center for Integrated Breeding Research,

Georg-August-University, Albrecht-Thaer-Weg 3, 37075 Göttingen, Germany.

Received: 14 April 2020 Accepted: 22 September 2020

Published online: 01 October 2020

\section{References}

1. Rodenburg TB, Buitenhuis AJ, Ask B, Uitdehaag KA, Koene P, van der Poel J, Bovenhuis $\mathrm{H}$. Heritability of feather pecking and open-field response of laying hens at two different ages. Poult Sci. 2003;82:861-7. https://doi.org/ 10.1093/ps/82.6.861.

2. Grams V, Wellmann R, Preuß S, Grashorn MA, Kjaer JB, Bessei W, Bennewitz J. Genetic parameters and signatures of selection in two divergent laying hen lines selected for feather pecking behaviour. Genet Sel Evol. 2015;47:77. https://doi.org/10.1186/s12711-015-0154-0.

3. Kjaer JB, Sørensen P. Feather pecking behaviour in white leghorns, a genetic study. Br Poult Sci. 1997;38:333-41. https://doi.org/10.1080/ 00071669708417999

4. Biscarini $F$, Bovenhuis $H$, van der Poel J, Rodenburg TB, Jungerius AP, van Arendonk JAM. Across-line SNP association study for direct and associative effects on feather damage in laying hens. Behav Genet. 2010;40:715-27. https://doi.org/10.1007/s10519-010-9370-0.

5. Su G, Kjaer JB, Sørensen P. Divergent selection on feather pecking behavior in laying hens has caused differences between lines in egg production, egg quality, and feed efficiency. Poult Sci. 2006;85:191-7. https://doi.org/10.1093/ ps/85.2.191.

6. Iffland H, Wellmann R, Schmid M, Preuß S, Tetens J, Bessei W, Bennewitz J. Genomewide Mapping of Selection Signatures and Genes for Extreme Feather Pecking in Two Divergently Selected Laying Hen Lines. Animals (Basel). 2020;10:262. https://doi.org/10.3390/ani10020262.

7. Buitenhuis AJ, Rodenburg TB, van Hierden YM, Siwek M, Cornelissen SJB, Nieuwland MGB, et al. Mapping quantitative trait loci affecting feather pecking behavior and stress response in laying hens. Poult Sci. 2003;82: 1215-22. https://doi.org/10.1093/ps/82.8.1215.

8. Lutz V, Stratz P, Preuß S, Tetens J, Grashorn MA, Bessei W, Bennewitz J. A genome-wide association study in a large F2-cross of laying hens reveals novel genomic regions associated with feather pecking and aggressive pecking behavior. Genet Sel Evol. 2017;49:18. https://doi.org/10.1186/ s12711-017-0287-4.

9. Flisikowski K, Schwarzenbacher $\mathrm{H}$, Wysocki M, Weigend S, Preisinger R, Kjaer $J B$, Fries R. Variation in neighbouring genes of the dopaminergic and serotonergic systems affects feather pecking behaviour of laying hens. Anim Genet. 2009:40:192-9. https://doi.org/10.1111/j.1365-2052.2008.01821.x.

10. Falker-Gieske C, Blaj I, Preuß S, Bennewitz J, Thaller G, Tetens J. GWAS for Meat and Carcass Traits Using Imputed Sequence Level Genotypes in Pooled F2-Designs in Pigs. G3 (Bethesda). 2019;9:2823-34. https://doi.org/10. 1534/g3.119.400452.

11. Blaj I, Tetens J, Preuß S, Bennewitz J, Thaller G. Genome-wide association studies and meta-analysis uncovers new candidate genes for growth and carcass traits in pigs. PLoS One. 2018;13:e0205576. https://doi.org/10.1371/ journal.pone.0205576.

12. Li Z, Chen J, Yu H, He L, Xu Y, Zhang D, et al. Genome-wide association analysis identifies 30 new susceptibility loci for schizophrenia. Nat Genet. 2017;49:1576-83. https://doi.org/10.1038/ng.3973.

13. Smeland OB, Wang Y, Frei O, Li W, Hibar DP, Franke B, et al. Genetic overlap between schizophrenia and volumes of Hippocampus, putamen, and intracranial volume indicates shared molecular genetic mechanisms. Schizophr Bull. 2018:44:854-64. https://doi.org/10.1093/schbul/sbx148.

14. Ikeda M, Takahashi A, Kamatani Y, Momozawa Y, Saito T, Kondo K, et al. Genome-wide association study detected novel susceptibility genes for schizophrenia and shared trans-populations/diseases genetic effect. Schizophr Bull. 2019:45:824-34. https://doi.org/10.1093/schbul/sby140.

15. Lam M, Chen C-Y, Li Z, Martin AR, Bryois J, Ma X, et al. Comparative genetic architectures of schizophrenia in east Asian and European populations. Nat Genet. 2019;51:1670-8. https://doi.org/10.1038/s41588-019-0512-x. 
16. Smeland OB, Bahrami S, Frei O, Shadrin A, O'Connell K, Savage J, et al. Genome-wide analysis reveals extensive genetic overlap between schizophrenia, bipolar disorder, and intelligence. Mol Psychiatry. 2019 https://doi.org/10.1038/s41380-018-0332-x.

17. Liu L, Wen Y, Ning Y, Li P, Cheng B, Cheng S, et al. A trans-ethnic two-stage polygenetic scoring analysis detects genetic correlation between osteoporosis and schizophrenia. Clin Transl Med. 2020;9:21. https://doi.org/ 10.1186/s40169-020-00272-y.

18. Iffland H, Wellmann R, Preuß S, Tetens J, Bessei W, Piepho H-P, Bennewitz J. A novel model to explain extreme feather pecking behavior in laying hens. Behav Genet. 2020;50:41-50. https://doi.org/10.1007/s10519-019-09971-w.

19. Hughes AL, Buitenhuis AJ. Reduced variance of gene expression at numerous loci in a population of chickens selected for high feather pecking. Poult Sci. 2010;89:1858-69. https://doi.org/10.3382/ps.2010-00827.

20. Bennewitz J, Bögelein S, Stratz P, Rodehutscord M, Piepho HP, Kjaer JB, Bessei W. Genetic parameters for feather pecking and aggressive behavior in a large F2-cross of laying hens using generalized linear mixed models. Poult Sci. 2014;93:810-7. https://doi.org/10.3382/ps.2013-03638.

21. Emrani $H$, Masoudi AA, Vaez TR, Ehsani A. Genome-wide association study of shank length and diameter at different developmental stages in chicken F2 resource population. Anim Genet. 2020. https://doi.org/10.1111/age.12981.

22. Sun Y, Li Q, Hu Y, Liu R, Zheng M, Wen J, et al. Genomewide association study of immune traits in chicken F2 resource population. J Anim Breed Genet. 2016. https://doi.org/10.1111/jbg.12186.

23. Yu G, Wang L-G, Han Y, He Q-Y. clusterProfiler: an R package for comparing biological themes among gene clusters. OMICS. 2012;16:284-7. https://doi. org/10.1089/omi.2011.0118.

24. Khan RAW, Chen J, Wang M, Li Z, Shen J, Wen Z, et al. A new risk locus in the ZEB2 gene for schizophrenia in the Han Chinese population. Prog Neuro-Psychopharmacol Biol Psychiatry. 2016;66:97-103. https://doi.org/10. 1016/j.pnpbp.2015.12.001.

25. Pillai S, Trevino J, Rawal B, Singh S, Kovacs M, Li X, et al. ß-arrestin-1 mediates nicotine-induced metastasis through E2F1 target genes that modulate epithelial-mesenchymal transition. Cancer Res. 2015;75:1009-20. https://doi.org/10.1158/0008-5472.CAN-14-0681.

26. Zhang S, Zhang X, Ma S, Purmann C, Davis K, Wong WH, et al. Network effects of the neuropsychiatric $15 q 13.3$ microdeletion on the transcriptome and epigenome in human induced neurons; 2019.

27. Alliey-Rodriguez N, Grey TA, Shafee R, Padmanabhan J, Tandon N, Klinger M, et al. Common variants of NRXN1, LRP1B and RORA are associated with increased ventricular volumes in psychosis - GWAS findings from the B-SNIP deep phenotyping study; 2017.

28. Chen H, Zhou Y, Ge Z, Li Q, Sun Q, Zheng L, et al. Association study of FGF18 with developmental dyslexia in Chinese population. Psychiatr Genet. 2018;28:8-11. https://doi.org/10.1097/YPG.0000000000000187.

29. Turner CA, Eren-Koçak E, Inui EG, Watson SJ, Akil H. Dysregulated fibroblast growth factor (FGF) signaling in neurological and psychiatric disorders. Semin Cell Dev Biol. 2016;53:136-43. https://doi.org/10.1016/j.semcdb.2015. 10.003.

30. Kaya $\mathrm{O}$. Investigation of the effects of nicotine on the expression profile of SW620 colon adenocarcinoma cells using afunctional genomics approach [Master's dissertation]. Ankara: Department of molecular biology and genetics; 2009.

31. Brunzell DH, Stafford AM, Dixon Cl. Nicotinic receptor contributions to smoking: insights from human studies and animal models. Curr Addict Rep. 2015;2:33-46. https://doi.org/10.1007/s40429-015-0042-2.

32. Falker-Gieske C, Mott A, Preuß S, Franzenburg S, Bessei W, Bennewitz J, Tetens J. Analysis of the brain transcriptome in lines of laying hens divergently selected for feather pecking. BMC Genomics. 2020. https://doi. org/10.1186/s12864-020-07002-1.

33. Kim H, McGrath BM, Silverstone PH. A review of the possible relevance of inositol and the phosphatidylinositol second messenger system (PI-cycle) to psychiatric disorders--focus on magnetic resonance spectroscopy (MRS) studies. Hum Psychopharmacol. 2005;20:309-26. https://doi.org/10.1002/ hup.693.

34. Rasika S, Passemard S, Verloes A, Gressens P, El Ghouzzi V. Golgipathies in neurodevelopment: a new view of old defects. Dev Neurosci. 2018;40:396416. https://doi.org/10.1159/000497035.

35. Martínez-Menárguez JÁ, Tomás M, Martínez-Martínez N, Martínez-Alonso E. Golgi fragmentation in neurodegenerative diseases: is there a common cause? Cells. 2019. https://doi.org/10.3390/cells8070748.
36. Opel N, Goltermann J, Hermesdorf M, Berger K, Baune BT, Dannlowski U. Cross-disorder analysis of brain structural abnormalities in six major psychiatric disorders: a secondary analysis of mega- and meta-analytical findings from the ENIGMA consortium. Biol Psychiatry. 2020. https://doi.org/ 10.1016/j.biopsych.2020.04.027.

37. Pardo M, Cheng Y, Sitbon YH, Lowell JA, Grieco SF, Worthen RJ, et al. Insulin growth factor 2 (IGF2) as an emergent target in psychiatric and neurological disorders. Rev Neurosci Res. 2019;149:1-13. https://doi.org/10.1016/j.neures. 2018.10.012.

38. Cheon S, Dean M, Chahrour M. The ubiquitin proteasome pathway in neuropsychiatric disorders. Neurobiol Learn Mem. 2019;165:106791. https:// doi.org/10.1016/j.nIm.2018.01.012.

39. Saus E, Brunet A, Armengol L, Alonso P, Crespo JM, Fernández-Aranda F, et al. Comprehensive copy number variant (CNV) analysis of neuronal pathways genes in psychiatric disorders identifies rare variants within patients. J Psychiatr Res. 2010;44:971-8. https://doi.org/10.1016/j.jpsychires.2010.03.007.

40. Castellani CA, Awamleh Z, Melka MG, O'Reilly RL, Singh SM. Copy number variation distribution in six monozygotic twin pairs discordant for schizophrenia. Twin Res Hum Genet. 2014;17:108-20. https://doi.org/10.1017/thg.2014.6.

41. Alblooshi H, Al Safar H, El Kashef A, Al Ghaferi H, Shawky M, Hulse GK, Tay GK. Stratified analyses of genome wide association study data reveal haplotypes for a candidate gene on chromosome 2 (KIAA1211L) is associated with opioid use in patients of Arabian descent. BMC Psychiatry. 2020;20:41. https://doi.org/10.1186/s12888-019-2425-8.

42. Moore JM, Oliver PL, Finelli MJ, Lee S, Lickiss T, Molnár Z, Davies KE. Laf4/ Aff3, a gene involved in intellectual disability, is required for cellular migration in the mouse cerebral cortex. PLoS One. 2014;9:e105933. https:// doi.org/10.1371/journal.pone.0105933.

43. Chen J, Tsang S-Y, Zhao C-Y, Pun FW, Yu Z, Mei L, et al. GABRB2 in schizophrenia and bipolar disorder: disease association, gene expression and clinical correlations. Biochem Soc Trans. 2009;37:1415-8. https://doi.org/ 10.1042/BST0371415.

44. Ng S-K, Lo W-S, Pun FW, Zhao C, Yu Z, Chen J, et al. A recombination hotspot in a schizophrenia-associated region of GABRB2. PLoS One. 2010;5: e9547. https://doi.org/10.1371/journal.pone.0009547.

45. Zhao C, Wang F, Pun FW, Mei L, Ren L, Yu Z, et al. Epigenetic regulation on GABRB2 isoforms expression: developmental variations and disruptions in psychotic disorders. Schizophr Res. 2012;134:260-6. https://doi.org/10.1016/j. schres.2011.11.029.

46. Tsang SY, Zhong S, Mei L, Chen J, Ng S-K, Pun FW, et al. Social cognitive role of schizophrenia candidate gene GABRB2. PLoS One. 2013;8:e62322. https://doi.org/10.1371/journal.pone.0062322.

47. Wang L, Jiang $W$, Lin $Q$, Zhang $Y$, Zhao C. DNA methylation regulates gabrb2 mRNA expression: developmental variations and disruptions in Imethionine-induced zebrafish with schizophrenia-like symptoms. Genes Brain Behav. 2016;15:702-10. https://doi.org/10.1111/gbb.12315.

48. Zhang $T$, Li J, Yu H, Shi Y, Li Z, Wang L, et al. Meta-analysis of GABRB2 polymorphisms and the risk of schizophrenia combined with GWAS data of the Han Chinese population and psychiatric genomics consortium. PLoS One. 2018;13:e0198690. https://doi.org/10.1371/journal.pone.0198690.

49. Yeung RK, Xiang Z-H, Tsang S-Y, Li R, Ho TYC, Li Q, et al. Gabrb2-knockout mice displayed schizophrenia-like and comorbid phenotypes with interneuron-astrocyte-microglia dysregulation. Transl Psychiatry. 2018;8:128. https://doi.org/10.1038/s41398-018-0176-9.

50. Zhang $Q$, Zhang $X$, Song $S$, Wang S, Wang $X, Y u$, et al. The association of GABRB2 SNPs with cognitive function in schizophrenia. Eur Arch Psychiatry Clin Neurosci. 2019. https://doi.org/10.1007/s00406-019-00985-3.

51. Kim YS, Yang M, Mat W-K, Tsang S-Y, Su Z, Jiang X, et al. GABRB2 haplotype association with heroin dependence in Chinese population. PLoS One. 2015;10:e0142049. https://doi.org/10.1371/journal.pone.0142049.

52. Kjaer JB, Sørensen P, Su G. Divergent selection on feather pecking behaviour in laying hens (Gallus gallus domesticus). Appl Anim Behav Sci. 2001;71:229-39. https://doi.org/10.1016/s0168-1591(00)00184-2.

53. Bessei W, Bauhaus H, Bögelein S. The effect of selection for high and low feather pecking on aggression - related behaviours of laying hens. Archiv für Geflügelkunde. 2013;77:10-4.

54. Savory CJ. Feather pecking and cannibalism. World's Poultry Sci J. 1995;51: 215-9. https://doi.org/10.1079/WPS19950016.

55. Piepho H-P, Lutz V, Kjaer JB, Grashorn M, Bennewitz J, Bessei W. The presence of extreme feather peckers in groups of laying hens. Animal. 2017; 11:500-6. https://doi.org/10.1017/S1751731116001579. 
56. McKenna A, Hanna M, Banks E, Sivachenko A, Cibulskis K, Kernytsky A, et al. The genome analysis toolkit: a MapReduce framework for analyzing nextgeneration DNA sequencing data. Genome Res. 2010;20:1297-303. https:// doi.org/10.1101/gr.107524.110.

57. Li H, Durbin R. Fast and accurate short read alignment with burrowswheeler transform. Bioinformatics. 2009;25:1754-60. https://doi.org/10.1093/ bioinformatics/btp324.

58. $\mathrm{Ni} \mathrm{G}$, Strom TM, Pausch $H$, Reimer C, Preisinger R, Simianer $H$, Erbe M. Comparison among three variant callers and assessment of the accuracy of imputation from SNP array data to whole-genome sequence level in chicken. BMC Genomics. 2015;16:824. https://doi.org/10.1186/s12864-0152059-2.

59. Willer CJ, Li Y, Abecasis GR. METAL: fast and efficient meta-analysis of genomewide association scans. Bioinformatics. 2010;26:2190-1. https://doi. org/10.1093/bioinformatics/btq340.

60. Carlson M. org. Gg.eg.db: Genome wide annotation for Chicken: R package; 2019.

61. Bessei W, Kjaer JB. Feather pecking in layers-state of research and implications. In: Poultry Research Foundation. Sydney: Australian Poultry Science Symposium; 2005.

\section{Publisher's Note}

Springer Nature remains neutral with regard to jurisdictional claims in published maps and institutional affiliations.

- fast, convenient online submission

- thorough peer review by experienced researchers in your field

- rapid publication on acceptance

- support for research data, including large and complex data types

- gold Open Access which fosters wider collaboration and increased citations

- maximum visibility for your research: over $100 \mathrm{M}$ website views per year

At $\mathrm{BMC}$, research is always in progress. 